\title{
USB-ENDOSCOPE LARYNGOSCOPE IS AS EFFECTIVE AS VIDEO LARYNGOSCOPE IN DIFFICULT INTUBATION
}

\author{
Bülent Güngörer ${ }^{1} \odot$, Meliha Findik², Afsin Emre Kayipmaz ${ }^{1}$ \\ ${ }^{1}$ Ankara City Hospital, Ankara, Turkey \\ ${ }^{2}$ Balikesir University Faculty of Medicine Department of Emergency Medicine, Balıkesir, Turkey
}

\begin{abstract}
BACKGROUND: Video laryngoscopy (VL) has recently been put into clinical use to minimize the limitations of direct laryngoscopy and assist physicians interested in airway management. However, the high cost is the biggest constraint especially in countries with limited resources. To lower the cost, a custom-made VL obtained by attaching a USB-endoscope camera $(\$ 8.5)$ to the Macintosh laryngoscope (USB-L) can be used.

MATERIAL AND METHODS: All intubations were performed in a difficult intubation model. Intubations were carried out by two emergency physicians. A Glidescope as a VL and a custom-made USB-L were used. In addition to these devices, one bougie to facilitate the advancement of the tube was used. The total intubation time was evaluated.
\end{abstract}

RESULTS: Correct tube placement for both operators was $100 \%$ for both devices. A difference between the operators in the duration of intubations could not be found. Also, there is no difference in the duration of intubations between the devices for both operators.

CONCLUSION: It was concluded that the USB-L and VL are not statistically different in terms of intubation time in the difficult intubation model using bougie. For countries with limited resources, the low-cost USB-L has come to the forefront due to the high cost of VL and difficulty of access.

KEY WORDS: difficult intubation, video laryngoscope, bougie, mannequin, intubation time

Disaster Emerg Med J 2021; 6(2): 75-79

\section{INTRODUCTION}

Direct laryngoscopy is a widely used method for endotracheal intubation in the emergency department. Video laryngoscopy has recently been put into clinical use to minimize the limitations of direct laryngoscopy especially in difficult intubation [1,2]. However, the high cost is the biggest constraint to this method's use, especially in emergency departments of countries with limited resources [3]. To overcome this situation, it has been suggested that a custom-made video laryngoscope obtained by attaching a universal serial bus (USB) endoscope camera to the Macintosh laryngoscope can be used for the same purpose [4]. It has been reported that this custom-made device (the USB-L) can assist in experimental studies and education of medical, paramedic students, and emergency medicine residents [5].

A study was carried out in India on the use of a custom-made laryngoscope in a clinical setting. The authors intubated half of 40 patients with similar age, gender, physical characteristics, American Society of Anesthesiologists, and Mallampati score with a Miller direct laryngoscope and bougie. The other half were intubated with a USB-L. There was no difference between the groups in terms of heart rate and mean arterial pressure after intubation. 
Although the mean of the total intubation times was shorter using the USB-L, there was no statistically significant difference between the two groups ( $p=0.712)[6]$.

It is shown that a USB-L provides similar vision, clarity, and ease of intubation to that of expensive video laryngoscopes [7]. The trials carried out by clinicians with this device will provide an idea for companies that can produce low-cost devices that can be connected to smartphones. In addition, during the COVID-19 pandemic, that custom-made devices like the USB-L will help protect healthcare professionals in high aerosol-forming processes in difficult intubation. However, there is no proof that USB-L is effective as a video laryngoscope in difficult intubation.

This study aimed to evaluate custom-made USB-endoscope laryngoscope effectiveness in difficult intubation compared to a well-known video laryngoscope.

\section{MATERIAL AND METHODS}

All intubations were performed using the AirSim Advance Bronchi $X$ intubation simulator model (mannequin) (TruCorp, Armagh, N. Ireland) and a difficult airway. Difficult AirSim Airway featured enlarged tongue oedema with an elongated notched epiglottis.

As the low-cost USB endoscope (\$8.5), a $5 \mathrm{~mm}$ diameter was used, waterproof, illuminated device compatible with Android, 480p and 0.3-megapixel resolution. Similar to previous studies, the USB endoscope was attached approximately $40 \mathrm{~mm}$ behind the tip of the traditional Macintosh direct laryngoscope (Fig. 2). The visual orientation was checked before attaching as described previously [5]. The USB camera was linked via a USB cable to the laptop and a micro-USB cable to the mobile device. The CameraFi (Vault Micro Inc., Seoul, Korea) software on the Android phone was used. Before each intubation, the USB camera and the cables were cleaned with alcohol-based medical device surface disinfectants.

We used a Glidescope-Titanium MAC T4 with a $60^{\circ}$ hyper angulated blade (Verathon, USA) as a reusable video laryngoscope. In addition to these devices, one bougie in both methods was used to facilitate the advancement of the tube.

Intubations were carried out by two emergency physicians experienced in intubation. The order of

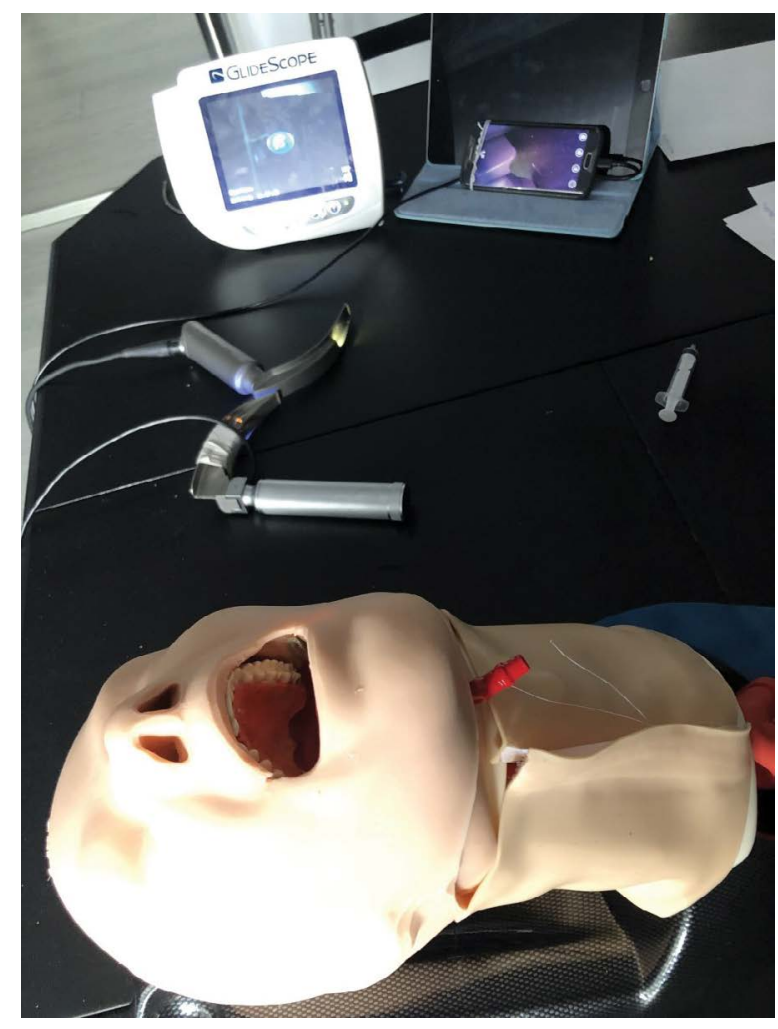

FIGURE 1. Demonstration of USB-L and video laryngoscope

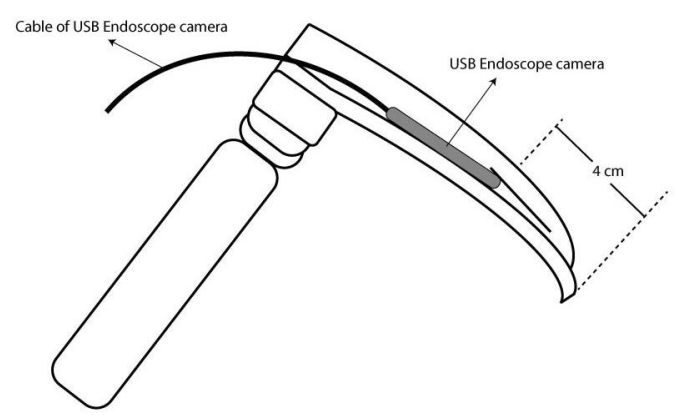

FIGURE 2. Schematic illustration of USB-L

difficult intubation with USB or video laryngoscope was randomized with rolling dice.

In the study, the total intubation time as the time from entering the laryngoscope blade in the mouth of the mannequin to placing the tube was evaluated. An emergency medicine professor checked all the processing steps as an independent observer.

\section{Statistical Analysis}

While calculating the sample size, Cohen's medium effect size was used because no predictions about the parameters to be investigated could be made, as no similar studies in the literature were found. Accordingly, as a result of the power analysis with $\mathrm{G}^{*}$ 
Power, when the effect width $d=0.50$ was taken, it was calculated that a total of at least 102 intubations would provide $80 \%$ test power and a $95 \%$ confidence level. Since two operators would intubate four times with two devices, it was found that each operator must perform at least 26 intubations with each device. Hereupon, each operator made 27 intubations.

Descriptive statistics like the mean were given [standard deviation (SD)], and intubation times were compared using a paired samples t-test. A p-value under 0.05 was accepted to be statistically significant. The statistical analysis using STATA 15.1 (Stata Corp., Collage Station, TX, USA) software was conducted.

\section{RESULTS}

The tracheal tube placement and glottic visualization succeeded in all intubations of the two operators for both devices. Operator 1 intubated the difficult intubation model in $26.97 \pm 26.98$ seconds via video laryngoscope and $20.10 \pm 6.53$ via USB-endoscope laryngoscope. Besides, operator 2 intubated the model in $22.83 \pm 10.52$ seconds via video laryngoscope and $18.96 \pm 6.11$ via USB-endoscope laryngoscope. Table 1 shows the number of intubation, the mean intubation times and standard deviation for Operator 1 and Operator 2.

In the comparison of intubation times of operator 1 and operator 2, both the video laryngoscope intubation time $(p=0.496)$ and the USB-endoscope laryngoscope intubation time $(p=0.542)$ was not different. Also, the intubation time via video laryngoscope or USB-endoscope laryngoscope was not different for both operators (Tab. 2).

\section{DISCUSSION}

This study could not find a statistically significant difference between the duration of intubations performed by both operators with different devices (video laryngoscope vs. USB-L by operator 1 and 2) individually. A statistically significant difference was not found between the duration of intubations performed by both operators using the same devices (video laryngoscope by operator 1 and 2 \& USB-L by operator 1 and 2), respectively. Based on this, it was concluded that the USB-L and video laryngoscope are not statistically different in terms of intubation time in the difficult intubation model using bougie.

Table 1. Mean intubation times of the two
operators in seconds
\begin{tabular}{|l|c|c|c|}
\hline & $\begin{array}{c}\text { Number of } \\
\text { intubation }\end{array}$ & $\begin{array}{c}\text { Number of } \\
\text { successful } \\
\text { intubation }\end{array}$ & $\begin{array}{c}\text { Mean } \pm \text { SD } \\
\text { (seconds) }\end{array}$ \\
\hline Op.1 VL & 27 & 27 & $26.97 \pm 26.98$ \\
\hline Op.1 USB-L & 27 & 27 & $20.10 \pm 6.53$ \\
\hline Op.2 VL & 27 & 27 & $22.83 \pm 10.52$ \\
\hline Op. 2 USB-L & 27 & 27 & $18.96 \pm 6.11$ \\
\hline
\end{tabular}

SD — Standard deviation; VL — video laryngoscope; USB-L — USB-endoscope laryngoscope

Table 2. Comparison of means of intubation times by Paired samples $t$-test

\begin{tabular}{|c|c|c|c|}
\hline & \multicolumn{2}{|c|}{ Paired differences } & \multirow[b]{2}{*}{$p$ value } \\
\hline & $\begin{array}{c}\text { Diff Mean } \pm \\
\text { SD }\end{array}$ & $\begin{array}{l}95 \% \text { Cls of } \\
\text { the difference }\end{array}$ & \\
\hline $\begin{array}{l}\text { Op. 1- VL vs. } \\
\text { Op. } 1 \text { - USB-L }\end{array}$ & $6.84 \pm 5.14$ & $-3.72-17.41$ & 0.195 \\
\hline $\begin{array}{l}\text { Op. 2- VL vs. } \\
\text { Op. 2- USB-L }\end{array}$ & $3.86 \pm 2.28$ & $-0.82-8.55$ & 0.102 \\
\hline $\begin{array}{l}\text { Op. 1- VL vs. } \\
\text { Op. 2- VL }\end{array}$ & $4.11 \pm 5.96$ & $-8.14-16.37$ & 0.496 \\
\hline $\begin{array}{l}\text { Op. 1- USB-L vs. } \\
\text { Op. 2- USB-L }\end{array}$ & $1.13 \pm 1.84$ & $-2.64-4.91$ & 0.542 \\
\hline
\end{tabular}

Op. - Operator; $\mathrm{Cl}$ - Confidence interval; SD — Standard deviation; VL — video laryngoscope; USB-L — USB-endoscope laryngoscope

Airway interventions are needed in these patients upon the development of the severe acute respiratory syndrome. However, tracheal intubation is a very high-risk method for airway management in these cases due to high levels of SARS-CoV-2 virus load in sputum and upper airway secretions. Therefore, appropriate precautions must be taken to keep personnel safe in the foreground. Although systematic research on healthcare workers' risk of infection is based on limited literature, it has again become prominent with COVID19 [8].

Guidelines recommend the use of an aerosol box covering a patient's head during tracheal intubation in the COVID-19 era. However, intubation can be challenging with protective equipment such as an aerosol box, eye goggles and face shield. Several expert recommendations have been made for the use of video laryngoscopes while intubating these patients so that the time required for intubation is minimized [9-12]. Currently, there are various video laryngoscopes in the market and it has been shown 
that they perform differently under these protective measures [13-14]. A study using stimulation of tracheal intubation in a patient with stimulation of COVID-19 showed prominent differences between six video laryngoscopes in patients with inhibited neck movement and limited mouth opening [13]. In a case series including adult patients that intubation with a Macintosh laryngoscope had been difficult, intubation with a video laryngoscope was successful in 290 of 293 patients [15]. Some other randomized, controlled studies comparing different video laryngoscopes have shown a higher success rate of intubation using the video laryngoscopes when compared to Macintosh laryngoscope [16-18]. It is now clear that video laryngoscopes increase the success rate of tracheal intubation with difficult airways.

In a previous study, the success rate of tracheal intubation in the first attempt was found to be around $80 \%$, and the risk of infection of health personnel was said to increase during multiple airway manipulations. For this reason, it is recommended to use airway techniques, such as a video laryngoscope, that are reliable and maximize success in the first attempt [8]. This study compared video laryngoscopy and USB-L. The placement of the tube took place in both methods with $100 \%$ accuracy. The authors believe that no significant difference between intubation times was detected because of the similar view and quality of USB-L and video laryngoscopy in intubation.

A study comparing normal and difficult airways with inexperienced operators evaluated the time and rate of successful intubation, the best view of the glottis, oesophageal intubation, dental trauma, and user satisfaction. As a result of this study, intubation-related times, glottis appearance, and operator satisfaction were found to be significantly higher in commercial video laryngoscopes. In contrast, custom-made video laryngoscopy performance was found to be similar to that of a Macintosh direct laryngoscope. As a result, it was found that video laryngoscopes are superior to Macintosh direct laryngoscopes in both normal and difficult airway scenarios for inexperienced users [19]. This study, due to the similar experience of both operators, did not present a significant difference between intubation times in similar devices (video laryngoscopy by operator 1 and 2 \& USB-L by operator 1 and 2).

Another study showed that the duration of intubation with the USB-L was shorter than that of the direct laryngoscope similar to this study. The duration of successful tracheal intubation was $26.92 \pm 5.03$ seconds in the USB-L group and $40.64 \pm 5.7$ seconds in the direct laryngoscopy group. This difference was considered statistically significant $(p<0.001)$. The authors also reported that the image quality of the USB-L may decrease due to condensation on the lens and secretion in the oral cavity [20]. Since the study was carried out on a mannequin, no imaging problems related to fogging on the lens and secretion were encountered.

In another study which investigated the success of USB-L in 50 elective surgery intubation, external laryngeal manipulation improved vision in $44 \%$ of intubations. Of the cases, $20 \%$ were intubated with the help of bougie, and the remaining cases were intubated directly with the endotracheal tube. The success rate on the first attempt of intubation was $82 \%$. The remaining $18 \%$ were intubated on the second attempt. For $64 \%$ of the intubations, the operators reported that intubation was easy and confident [21]. In this study, both operators used USB-L with bougie for all difficult intubations. In no attempts were the endotracheal tubes located in the oesophagus. Furthermore, no difficulty was encountered in guiding and advancing the endotracheal tube in the use of video laryngoscopes in this method. The bougie enabled the tube to move easily through the airway. Unlike in that study, external laryngeal manipulation was not needed, as intubations were not performed on the patients.

In the COVID-19 pandemic, it has been reported that the use of video laryngoscopy is a safer method for healthcare workers, as tracheal intubations are considered very high risk in terms of contamination [8]. During the pandemic, the use of low-cost devices that will be attached to the USB camera instead of a direct laryngoscope has also come into question, especially in a limited resource setting.

\section{CONCLUSIONS}

The authors concluded that the USB-L and video laryngoscope are not statistically different in terms of intubation time in the difficult intubation model using bougie. For countries with limited resources, the low-cost USB-L has come to the forefront due to the high cost of video laryngoscopes and the difficulty of access. 


\section{REFERENCES}

1. Maslanka M, Smereka J, Czyzewski L, et al. VieScope $®$ laryngoscope versus Macintosh laryngoscope during difficult intubation performed by paramedics: a randomized cross-over manikin trial. Disaster and Emergency Medicine Journal. 2020, doi: 10.5603/demj.a2020.0031.

2. Sip M, Zalewski R, Klosiewicz T, et al. Use of video laryngoscopes by inexperienced personnel in difficult intubations. Disaster and Emergency Medicine Journal. 2018; 3(3): 96-100, doi: 10.5603/demj.2018.0021.

3. Zaouter C, Calderon J, Hemmerling TM. Videolaryngoscopy as a new standard of care. Br J Anaesth. 2015; 114(2): 181-183, doi: 10.1093/ bja/aeu266, indexed in Pubmed: 25150988.

4. Karippacheril JG, Umesh G, Nanda S. Assessment and confirmation of tracheal intubation when capnography fails: a novel use for an USB camera. J Clin Monit Comput. 2013; 27(5): 531-533, doi: 10.1007/ s10877-013-9458-1, indexed in Pubmed: 23536203.

5. Trivedi JN. An economical model for mastering the art of intubation with different video laryngoscopes. Indian J Anaesth. 2014; 58(4): 394-396, doi: 10.4103/0019-5049.138967, indexed in Pubmed: 25197105.

6. Vadhanan P, Balakrishnan K, Tripaty DK. Evaluation of a low-cost videolaryngoscope - A randomized controlled pilot study. Anaesthesia, Pain Intensive Care. 2017; 21(4): 406-12.

7. Hasija N, Kale S, Girdhar KK. Digitizing the direct laryngoscopy experience: the economic way!! Korean J Anesthesiol. 2018; 71(6): 486-487, doi: 10.4097/kja.d.18.00093, indexed in Pubmed: 29760368.

8. Cook TM, El-Boghdadly K, McGuire B, et al. Consensus guidelines for managing the airway in patients with COVID-19: Guidelines from the Difficult Airway Society, the Association of Anaesthetists the Intensive Care Society, the Faculty of Intensive Care Medicine and the Royal College of Anaesthetists. Anaesthesia. 2020; 75(6): 785-799, doi: 10.1111/anae.15054, indexed in Pubmed: 32221970.

9. Yao $W$, Wang $T$, Jiang $B$, et al. collaborators. Emergency tracheal intubation in 202 patients with COVID-19 in Wuhan, China: lessons learnt and international expert recommendations. Br J Anaesth. 2020; 125(1): e28-e37, doi: 10.1016/j.bja.2020.03.026, indexed in Pubmed: 32312571.

10. Lai HY. Aerosol Box. Protects healthcare providers during endotracheal intubation. Available from: https://sites.google. com/view/aerosolbox/ home (accessed 18 Feb. ; 2021.

11. Canelli R, Connor CW, Gonzalez M, et al. Barrier Enclosure during Endotracheal Intubation. N Engl J Med. 2020; 382(20): 1957-1958, doi: 10.1056/NEJMc2007589, indexed in Pubmed: 32243118.
12. Cook TM, El-Boghdadly K, McGuire $B$, et al. Consensus guidelines for managing the airway in patients with COVID-19. Anaesthesia. 2020; 75(6): 785-799, doi: 10.1111/anae.15054.

13. Kleine-Brueggeney $M$, Greif $R$, Schoettker $P$, et al. Evaluation of six videolaryngoscopes in 720 patients with a simulated difficult airway: a multicentre randomized controlled trial. Br J Anaesth. 2016; 116(5): 670-679, doi: 10.1093/bja/aew058, indexed in Pubmed: 27106971.

14. Asai T. Videolaryngoscopes: do they truly have roles in difficult airways? Anesthesiology. 2012; 116(3): 515-517, doi: 10.1097/ ALN.0b013e318246e866, indexed in Pubmed: 22261794.

15. Asai T, Liu EH, Matsumoto S, et al. Use of the Pentax-AWS in 293 patients with difficult airways. Anesthesiology. 2009; 110(4): 898-904, doi: 10.1097/ALN.0b013e31819c45e5, indexed in Pubmed: 19293707.

16. Jungbauer $A$, Schumann $M$, Brunkhorst $V$, et al. Expected difficult tracheal intubation: a prospective comparison of direct laryngoscopy and video laryngoscopy in 200 patients. Br J Anaesth. 2009; 102(4): 546-550, doi: 10.1093/bja/aep013, indexed in Pubmed: 19233881.

17. Malik MA, Subramaniam $\mathrm{R}$, Maharaj $\mathrm{CH}$, et al. Randomized controlled trial of the Pentax AWS, Glidescope, and Macintosh laryngoscopes in predicted difficult intubation. Br J Anaesth. 2009; 103(5): 761-768, doi: 10.1093/bja/aep266, indexed in Pubmed: 19783539.

18. Enomoto $Y$, Asai T, Arai $T$, et al. Pentax-AWS, a new videolaryngoscope, is more effective than the Macintosh laryngoscope for tracheal intubation in patients with restricted neck movements: A randomized comparative study. Br J Anaesth. 2008; 100: 544-8.

19. Rendeki S, Keresztes D, Woth G, et al. Comparison of VividTrac ${ }^{\circledR}$, Airtraq ${ }^{\circledR}$, King Vision ${ }^{\circledR}$, Macintosh Laryngoscope and a Custom-Made Videolaryngoscope for difficult and normal airways in mannequins by novices. BMC Anesthesiol. 2017; 17(1): 68, doi: 10.1186/s12871017-0362-y, indexed in Pubmed: 28549421.

20. LIU Hu, SHI Xy, CHEN W, et al. Comparison between HPHJ-A video laryngoscope and macintosh laryngoscope in clinical intubation. Academic Journal of Second Military Medical University. 2010; 30(10): 1073-1075, doi: 10.3724/sp.j.1008.2010.01073.

21. Narayan AK, Janardhan AL, Prakash A, et al. Low-cost teaching aid: A modification of Macintosh blade into a video laryngoscope for teaching laryngoscopy and intubation. J Anaesthesiol Clin Pharmacol. 2018; 34(4): 552-554, doi: 10.4103/joacp.JOACP_337_17, indexed in Pubmed: 30774244. 